\title{
Varicella Vaccine: What Have You Done for Me Lately?
}

Anne A. Gershon, MD

The live attenuated varicella vaccine remains the only vaccine in use against a virus that has a latent form and therefore causes 2 illnesses: varicella and zoster (also known as shingles). ${ }^{1}$ Zoster occurs after latent infection with varicella zoster virus (VZV) reactivates, after either chickenpox or vaccination. Viral latency lasts a lifetime. In countries where the vaccine is not used, varicella is mainly a disease of children, and zoster is a disease of adults. Because of declining cell-mediated immunity in older individuals, the incidence of zoster increases sharply after age 50. However, zoster may occur at any age in individuals who have previously been infected with VZV, and children vaccinated against chickenpox are at risk. In addition to waning cellular immunity with age, other factors that may precipitate zoster include immunosuppression, other viral infections, and stress. ${ }^{1}$

The varicella vaccine was developed in Japan in 1974, after Takahashi et $\mathrm{al}^{2}$ successfully attenuated VZV that was obtained from an otherwise healthy child with chickenpox. When the varicella vaccine was initially tested in the United States in the late 1970s, children with leukemia were frequently on their way to being cured of leukemia, only to succumb to overwhelming varicella because they were immunosuppressed. Therefore, in the late 1970s, vaccination of US children with leukemia was undertaken to try to prevent varicella because they had a positive risk/benefit ratio in comparison with healthy children in whom varicella was usually mild. It was found that the varicella vaccine protected children with leukemia not only from chickenpox but also from zoster. ${ }^{3,4}$ It was possible to obtain these observations within a short time limit; the attack rates of both varicella and zoster were then high in these children because they were immunocompromised. The varicella vaccine was found to be both effective and safe in preventing VZV infections in children with leukemia in remission, which paved the way for vaccination of varicella in healthy children.

It was subsequently determined that varicella vaccination also decreased the incidence, morbidity, and mortality from varicella in healthy children, with 2 doses being superior to 1 dose. ${ }^{5}$ It proved more difficult to determine if the varicella vaccine offered protection against zoster, which involves a more complex analysis.

In the article by Weinmann et al, $^{6}$ it was necessary to evaluate several groups of healthy children who were vaccinated at different ages to determine if varicella vaccine protected against zoster. Children are usually immunized at age 1 ; after that age, some children may or may not have received the varicella vaccine. Whereas young children who are unvaccinated are not at risk to develop zoster because they are free of latent infection, this is not the case for children who are vaccinated. Comparing the 2 groups initially suggested that vaccination against chickenpox led to an increase in zoster. Only after there was a substantial number of children who were not vaccinated (and who came
Department of Pediatrics, Vagelos College of Physicians and Surgeons, Columbia University, New York, New York

Opinions expressed in these commentaries are those of the authors and not necessarily those of the American Academy of Pediatrics or its Committees.

DOI: https://doi.org/10.1542/peds.2018-3561

Accepted for publication Mar 20, 2019

Address correspondence to Anne A. Gershon, MD, Department of Pediatrics, Columbia University Vagelos College of Physicians and Surgeons, $630 \mathrm{~W}$ 168th St, New York, NY 10032. E-mail: aag1@ cumc.columbia.edu

PEDIATRICS (ISSN Numbers: Print, 0031-4005; Online, 1098-4275).

Copyright (C) 2019 by the American Academy of Pediatrics

FINANCIAL DISCLOSURE: Other than those listed under the Potential Conflict of Interest, the author has indicated she has no financial relationships relevant to this article to disclose.

FUNDING: Funded by the National Institutes of Health (R01 DK093094). Funded by the National Institutes of Health (NIH)

POTENTIAL CONFLICT OF INTEREST: Dr Gershon's laboratory has been supported by Merck by a service contract examining the safety of varicella zoster virus vaccines. This activity was required by the US Food and Drug Administration and involves molecular laboratory procedures on submitted specimens. There is and was no salary support or other payment to Dr Gershon. Dr Gershon has also consulted for Saol Therapeutics on varicella zoster immune globulin use.

COMPANION PAPER: A companion to this article can be found online at www.pediatrics.org/cgi/doi/10. 1542/peds.2018-2917.

To cite: Gershon AA. Varicella Vaccine: What Have You Done for Me Lately?. Pediatrics. 2019; 144(1):e20183561 
down with chickenpox) to compare with vaccine recipients could it be determined if vaccination protected against zoster compared with natural chickenpox. Eventually, it became clear that children who are vaccinated had a lower incidence of zoster than children who are unvaccinated. Note that children who are unvaccinated and developed zoster must have experienced chickenpox followed by latent infection to manifest a reactivation of wild-type VZV.

The remarkable observation in the article by Weinmann et $\mathrm{al}^{6}$ is that after reviewing the records of $>6$ million children between 2003 and 2014 by using International Classification of Diseases, Ninth Revision codes, it was found that the incidence of zoster in children who were vaccinated was $78 \%$ lower than in children who were unvaccinated. This indicated that the Oka vaccine was less likely to reactivate than wildtype VZV. The vaccine was even better at preventing zoster when 2 vaccine doses were given. That the varicella vaccine prevents not only varicella but zoster as well is an exciting dual benefit from the varicella vaccine, further improving the health of children by immunization. Additional studies will be necessary to show the mechanism for the protection against zoster (viral, immunologic, or both), how long this benefit lasts, and whether additional doses of some form of VZV vaccine will be more useful. It is possible that newer noninfectious zoster vaccines may be introduced for young adults at some future time.

Of concern are the children who may not be vaccinated in an era of vaccine hesitancy when clinical varicella is becoming uncommon. Exposure to varicella or zoster can result in clinical varicella in people who are susceptible. Clinical varicella is worse in adulthood than in childhood. Efforts to immunize all children against chickenpox must continue to be made to protect our population from wild-type VZV. Fortunately, antiviral therapy is also available for individuals who are unvaccinated and develop varicella or zoster, but immunization is, as usual, preferable.

\section{ABBREVIATION}

VZV: varicella zoster virus

\section{REFERENCES}

1. Gershon AA, Breuer J, Cohen Jl, et al. Varicella zoster virus infection. Nat Rev Dis Primers. 2015;1:15016

2. Takahashi M, Otsuka T, Okuno Y, Asano Y, Yazaki T. Live vaccine used to prevent the spread of varicella in children in hospital. Lancet. 1974;2(7892): 1288-1290

3. Gershon AA, Steinberg SP, Gelb L, et al Live attenuated varicella vaccine. Efficacy for children with leukemia in remission. JAMA. 1984;252(3):355-362

4. Hardy I, Gershon AA, Steinberg SP, LaRussa P. The incidence of zoster after immunization with live attenuated varicella vaccine. A study in children with leukemia. Varicella Vaccine Collaborative Study Group. N Engl J Med. 1991;325(22):1545-1550

5. Marin M, Güris D, Chaves SS, Schmid S, Seward JF; Advisory Committee on Immunization Practices, Centers for Disease Control and Prevention (CDC). Prevention of varicella: recommendations of the Advisory Committee on Immunization Practices (ACIP). MMWR Recomm Rep. 2007; 56(RR-4): $1-40$

6. Weinmann S, Naleway AL, Koppolu P, et al. Incidence of herpes zoster among children: 2003-2014. Pediatrics. 2018; 144(1):e20182917 